\title{
A Joint Multiresponse Split-Plot Modeling and Optimization Including Fixed and Random Effects
}

\author{
Rossella Berni \\ University of Florence
}

\author{
Nedka Dechkova Nikiforova \\ University of Florence
}

\begin{abstract}
This paper deals with a proposal for joint modeling and process optimization for splitplot designs analyzed through mixed response surface models. It addresses the following main issues: i) the building of a joint mixed response surface model for a multiple response situation, by defining only one response through which specific coefficients are included for studying the association among the responses; ii) the considering of fixed as well as random effects within a joint modeling and optimization context; iii) the achievement of an optimal solution by involving specific as well as common coefficients for the responses. We illustrate our contribution through a case-study related to a split-plot design on electronic components of printed circuit boards (PCBs); we obtain satisfactory results by confirming the validity of this contribution, where the qualitative factor PCB is also studied and optimized.
\end{abstract}

Keywords: experimental design, mixed response surface model, robust design approach.

\section{Introduction}

Since 1990s, split-plot design has received great attention as a valid plan in the technological field for a robust design approach (Box and Jones 1992). Interesting literature has recently been published on this experimental design and related topics in process optimization. Undoubtedly, the particular structure of split-plot design, e.g. bi-randomization, helps to solve some problems, such as the well-known differentiation among hard-to-change factors and easyto change factors (Logothetis and Wynn 1989) in a Response Surface Methodology (RSM) setting (Myers and Montgomery 2002). In connection with these issues, split-plot design is one of the fundamental designs when noises and/or random factors are present. Therefore, the latest developments on split-plot design in a robust design framework are attributable, inter alia, to Robinson, Pintar, Anderson-Cook, and Hamada (2012) and Tan and Wu (2013), where two Bayesian approaches are introduced in order to apply the split-plot in a robust design context and also evaluate non-normally distributed response variables. Nevertheless, a further challenge is the consideration of the multiple response case starting from the seminal papers of Derringer and Suich (1980) and Khuri and Conlon (1981). In this direction, many authors have contributed towards improving this relevant topic in order to solve the problem of finding an unique solution which could be an optimal compromise following process issues, 
noises, weighting and computational aspects (Vining and Myers 1990; Lin and Tu 1995; Tang and Xu 2002; Del Castillo 2007). Recently, further studies have attempted to solve this problem by considering both data analysis and external or subjective decisions (Lee, Kim, and Köksalan 2012); or by defining a Bayesian model-averaging approach based on building of a loss function ( $\mathrm{Ng} 2010)$.

Robust parameter design in a multiresponse context is also studied through the noise setting, such as in Bingham and Nair (2012) and Del Castillo, Colosimo, and Alshraideh (2012), where the proposal relates to the optimization of functional response profiles in a dynamic response setting.

When considering all the issues and features raised here, e.g. a split-plot design in a robust design context and a multiresponse process optimization, it becomes relevant to introduce a mixed model analysis (Khuri 1996, 2006; Khuri and Mukhopadhyay 2010), in which random as well as fixed variables and effects are estimated (Krueger and Montgomery 2014). With this line of reasoning, some developments have been introduced to solve the problem of ordinal responses (Goos and Gilmour 2012). Furthermore, in a mixed model approach for a split-plot design, Ordinary Least Squares (OLS) and Generalized Least Squares (GLS) estimates of fixed effects are equivalent when specific conditions are met (Vining, Kowalski, and Montgomery 2005). Further developments in this direction are related to the building of optimal split-plot designs by also evaluating the relevance of variance components and the Restricted Maximum Likelihoood (REML) estimation method (Mylona, Goos, and Jones 2014; Jones and Goos 2012). In this direction, research developments are introduced for improving statistical inference through split-plot or multi-stratum designs (Trinca and Gilmour 2017), or for detecting the Pure Error (and Lack-of-Fit test) in the presence of blocked variables or multi-stratum designs (Goos and Gilmour 2017).

This way, Lin (2018) defined an optimal (and robust) split-plot design in order to overcome problems of model misspecification due to the presence of aliasing effects and/or when potential model terms are missing. Furthermore, Berni (2012) applied a joint mixed response surface model and optimization for a split-plot design in the multiple response case; Ivanova, Molenberghs, and Verbeke (2019) illustrated a similar proposal, although by considering different contexts and aims.

The most recent developments are related to the use of experimental design, e.g. split-plot designs and non-linear mixed models, in a reliability context (Medlin, Freeman, Kensler, and Vining 2019).

In this paper, the main purpose is to propose a joint model for a split-plot design where several responses are studied through the building of only one response variable, which also allows for detecting the relation among them. In particular, the joint model is built within the mixed Response Surface (RS) context in order to study a robust process optimization. The defined joint multiresponse split-plot model allows us to solve the following four main issues: i) starting from a multiple response case, only one response variable is built, and then only one joint statistical model is estimated; ii) specific coefficients in the joint statistical model are explicitly defined in order to analyse the contribution of each response variable within the joint statistical model; iii) variance and covariance parameters are jointly evaluated by considering the specific sources of variability involved in the split-plot design, also conditioned to each response variable; iv) the robust process optimization is carried out through the joint estimated statistical model, and by also considering fixed as well as random effects. The latter allows us to significantly improve the optimization analytical procedure. Moreover, by involving all the responses, the joint modeling not only allows us to study specific estimates and effects for each response, but also to consider common estimated coefficients for analyzing peculiar characterizations among responses.

It must be noted that we estimate only one model regardless of the number of responses, and this improvement allows for a single optimization procedure. Furthermore, the subsequently simultaneous optimization step involves just one objective function in which each estimated 
response surface is considered through the defined joint model. The objective function is implemented by also considering the different contributions of the fixed and random effects. We illustrate the suggested theory through a split-plot case-study in the microelectronics field, where the analysis of the soldering performance of electronic components on printed circuit boards is carried out according to the specific materials used.

The paper is organized as follows: in Section 2 the theory related to the definition of the mixed split-plot model and joint modeling is outlined; in particular, the proposal is advanced for joint modeling and optimization of several response variables. Section 3 reports the casestudy, related to the description of the split-plot design in the multiple response case, aimed at illustrating the theory; the final remarks follow in Section 4.

\section{Outlined theory and the joint mixed split-plot modeling proposal}

This section illustrates the proposal for a joint mixed RS modeling and optimization. The linear mixed-effect model is illustrated in Section 2.1, while the general split-plot model and the mixed split-plot model are described in Sections 2.2 and 2.3 respectively; the proposal for the joint mixed split-plot modeling and the optimization procedure are presented in Sections 2.4 and 2.5 respectively; lastly, a summary of the full suggested procedure is reported in Section 2.6.

\subsection{The linear mixed-effect model}

The starting point is the consideration of a general linear mixed-effect model (Searle, Casella, and McCulloch 1992), as in formula (1), which is an extension of the general linear model that allows a more flexible structure for the specification of the covariance matrix of the random error. In other words, it allows for both correlation and heterogeneous variances, although normality is always assumed.

$$
\boldsymbol{y}=\boldsymbol{X} \boldsymbol{\beta}+\boldsymbol{Z} \boldsymbol{\gamma}+\boldsymbol{\epsilon}
$$

Formula (1) refers to a general response variable $\boldsymbol{y}$ of dimension $[N \times 1] ; \boldsymbol{X}$ is the matrix for fixed effects with dimension $[N \times p] ; \boldsymbol{\beta}$ is the column vector of the unknown coefficients for the fixed effects and it has dimension $[p \times 1]$. The matrix $\boldsymbol{Z}$ and the column vector $\boldsymbol{\gamma}$ are related to random effects, and they have dimensions $[N \times h]$ and $[h \times 1]$ respectively; finally $\epsilon$ is the column vector $[N \times 1]$ for the random error. It must be noted that the main difference with respect to the general linear model is the addition of the known design matrix $\boldsymbol{Z}$, and the vector of unknown random-effect parameters, $\boldsymbol{\gamma}$. In general, the matrix $\boldsymbol{Z}$ can contain either continuous or dummy variables, just like the array $\boldsymbol{X}$. The name mixed model derives from the fact that the model contains both fixed-effect coefficients $\boldsymbol{\beta}$, and random-effect coefficients $\gamma$.

In this context, the variance of the response variable $\boldsymbol{y}$ is modelled through the random-effect design matrix $\boldsymbol{Z}$ and by specifying the covariance structures of two matrices: i) the matrix $\boldsymbol{G}$ related to the estimates of variance-covariance for random effects; and ii) the matrix $\boldsymbol{R}$ of variance-covariance estimates for the random error. Thus, the matrix $\boldsymbol{V}$ of variance-covariance for $\boldsymbol{y}$ has the following structure:

$$
V=Z G Z^{\prime}+R
$$

where $\boldsymbol{V}$ is decomposed into two terms: a first term $\boldsymbol{Z} G \boldsymbol{Z}^{\prime}$ relating to the random effects and a second term, the matrix $\boldsymbol{R}$, relating to the variance-covariances of the error estimates.

Variance and covariances for random effects ( $\boldsymbol{G}$ and $\boldsymbol{R}$ arrays) are estimated through the Restricted Maximum Likelihood-REML method. To this end, the log-likelihood function $\log L(\boldsymbol{G}, \boldsymbol{R})$ for the REML equation is defined as follows:

$$
\log L(\boldsymbol{G}, \boldsymbol{R})=-\frac{1}{2} \log |\boldsymbol{V}|-\frac{1}{2} \log \left|\boldsymbol{X}^{\prime} \boldsymbol{V}^{-1} \boldsymbol{X}\right|-\frac{1}{2}(\boldsymbol{y}-\boldsymbol{X} \hat{\beta})^{\prime} \boldsymbol{V}^{-1}(\boldsymbol{y}-\boldsymbol{X} \hat{\beta})
$$


where the term $(\boldsymbol{y}-\boldsymbol{X} \hat{\boldsymbol{\beta}})^{\prime} \boldsymbol{V}^{-1}(\boldsymbol{y}-\boldsymbol{X} \hat{\boldsymbol{\beta}})$ is the expression to perform the Generalized Least Squares (GLS) estimation for the fixed effects, for further details see Searle et al. (1992).

\subsection{The split-plot model}

A split-plot design is characterized by a peculiar structure, based on the distinction of the experimental factors in two sets: the Whole-Plot (WP) and the Sub-Plot (SP) factors. A bi-randomization framework characterizes this distinction between WP and SP factors, and it implies the generation of two types of experimental units, e.g. WP units and SP units, with two corresponding error terms (Logothetis and Wynn 1989; Vining et al. 2005). More precisely, firstly the WP factors are randomly assigned to the WP experimental units, by generating the whole-plot error term; subsequently the SP factors are randomly assigned within each WP unit, by generating the SP error term. The split-plot design became fundamental, starting from the seminal contribution of Box and Jones (1992), in industrial experimentation and for the robust design approach. In fact, the peculiar structure of the split-plot design is particularly suitable in the technological field when considering specific issues both related to the product and/or the process to be improved, and the conduction of the experimental trials.

In general, let us define the set $Z=\left\{Z_{1}, . ., Z_{I}\right\}$ of WP factors, and the set $X=\left\{X_{1}, . ., X_{J}\right\}$ of SP factors that define the entire experimental region $\chi$. For each replicate $\boldsymbol{b}_{k}, k=1, . ., K$, and for the balanced case, we have $n$ runs, and therefore, the total number of trials is $N=n \times K$; $b_{u k}$ is the $u$-th value of the replicate vector $\boldsymbol{b}_{k}=\left(b_{1 k}, . ., b_{n k}\right)$. Furthermore, for each $k-t h$ replicate, we define $\boldsymbol{z}_{i}=\left(z_{i 1}, . ., z_{i u}, . ., z_{i n}\right)$ as the generic column vector, related to the $i$-th whole-plot factor, $(i=1, . ., I)$; while $\boldsymbol{x}_{j}=\left(x_{j 1}, . ., x_{j u}, . ., x_{j n}\right)$ is the general column vector for the $j$-th sub-plot factor.

In addition, we consider the case of a split-plot model as a $2^{\text {nd }}$ order model in a mixed RS setting (Khuri 1996). The mixed split-plot RS model, defined for $I$ whole-plot variables, $J$ sub-plot variables, a single replicate $(K=1)$, a single response variable $y$, and for a single observation $u,(u=1, . ., n)$, can be written as:

$$
\begin{aligned}
y_{u}(Z, X)= & \beta_{0}+\sum_{i=1}^{I} \gamma_{i} z_{i u}+\sum_{i=1 ; i<i^{\prime}}^{I-1} \sum_{i^{\prime}=i+1}^{I} \gamma_{i i^{\prime}} z_{i u} z_{i^{\prime} u}+\sum_{i=1}^{I} \gamma_{i i} z_{i u} z_{i u}+ \\
& \sum_{j=1}^{J} \beta_{j} x_{j u}+\sum_{j=1 ; j<j^{\prime}}^{J-1} \sum_{j^{\prime}=j+1}^{J} \beta_{j j^{\prime}} x_{j u} x_{j^{\prime} u}+\sum_{j=1}^{J} \beta_{j j} x_{j u} x_{j u}+ \\
& \sum_{i=1}^{I} \sum_{j=1}^{J} \delta_{i j} z_{i u} x_{j u}+\psi_{u}+\epsilon_{u}
\end{aligned}
$$

Note that $\gamma_{i}, \gamma_{i i^{\prime}}$ and $\gamma_{i i}$ are the random coefficients related to the WP variables, main effects, first order interactions and quadratic effects; $\beta_{0}$ and $\beta_{j}$ are the coefficients of the intercept and the fixed main-effects for the SP variables; while $\beta_{j j^{\prime}}$ and $\beta_{j j}$ are coefficients for the first order interactions and quadratic terms respectively. The random parameters $\delta_{i j}$ are the interaction effects between WP and SP factors, and they are specifically defined for evaluating the robust modeling approach.

The error terms are represented by $\psi_{u}$ (whole-plot error) and $\epsilon_{u}$ (sub-plot error). In general, $\psi_{u}$ and $\epsilon_{u}$ are supposedly independent and identically normally distributed, e.g. $\boldsymbol{\psi} \sim$ i.i.d. $N\left(0, \sigma_{\psi}^{2}\right)$ and $\boldsymbol{\epsilon} \sim$ i.i.d. $N\left(0, \sigma_{\epsilon}^{2}\right)$. In this initial step, we maintain this assumption; in what follows, we also consider different situations, even though the normality assumption still remains valid. It must be noted that when considering $K$ replicates $(K \geq 2)$, the replicate effect is included in the model and estimated through the matrix $\boldsymbol{R}$, as illustrated in details in the case-study.

In what follows, the split-plot model in a mixed RS model setting is reported in details. 


\subsection{The mixed split-plot model}

When considering the multiple response case and the general split-plot model (4), the mixed linear model (1) for a single $t$-th response variable $\boldsymbol{y}_{t}(t=1, . ., T)$ may be also formulated as in the following:

$$
\begin{aligned}
\boldsymbol{y}_{t}(Z, X) & =\boldsymbol{X}_{t} \boldsymbol{\beta}_{t}+\boldsymbol{Z}_{t} \gamma_{t}+\boldsymbol{\Delta}_{t} \boldsymbol{\delta}_{t}+\boldsymbol{\epsilon}_{t} \\
& =\boldsymbol{X}_{t} \boldsymbol{\beta}_{t}+\dot{\boldsymbol{Z}}_{t} \dot{\gamma}_{t}+\boldsymbol{\epsilon}_{t}
\end{aligned}
$$

where the set of experimental variables, e.g. WP and SP factors involved in the split-plot design, are related to random and fixed effects for the robust design setting respectively. Therefore, in a general situation, we consider that the matrix $\boldsymbol{X}_{t}$ and the vector $\boldsymbol{\beta}_{t}$ are related to the fixed effects for the SP variables. Furthermore, the matrix $\dot{\boldsymbol{Z}}$ is formed by the horizontal concatenation of the following two matrices:

- the matrix $\boldsymbol{Z}_{t}$ related to the random effects for the WP factors (formula (5)) (e.g. related to the main effects $\gamma_{i}$, the first order interactions $\gamma_{i i^{\prime}}$ and the quadratics effects $\gamma_{i i}$, formula (4));

- the matrix $\boldsymbol{\Delta}_{t}$ related to the interactions among WP and SP variables (formula (5)) (e.g. the crossed terms $z_{i u} x_{j u}$, formula (4)).

Similarly, the vector $\dot{\gamma}_{t}$ in formula (6) is formed by:

- the vector $\gamma_{t}$ that includes the random effects for the WP factors (formula (5));

- the vector $\boldsymbol{\delta}_{t}$ related to the random coefficients for the crossed terms among the WP and SP variables (formula (5)), these model terms play a relevant role for the robust design approach.

It must also be noted that in formulas (5) and (6) we consider a general structure of the split-plot model, in which the WP factors are considered as random effects. In the case-study the WP factors are treated accordingly to the empirical issues, as afterwards outlined (Section $3)$.

Moreover, by considering a single response variable $\boldsymbol{y}$ in a mixed RS model approach, the two error components $\psi_{u}$ and $\epsilon_{u}$ of a general split-plot model (4) are estimated through the $\boldsymbol{G}$ and $\boldsymbol{R}$ matrices, formula (2); the WP error $\psi_{u}$ (model (4)) is then absorbed within the random structure of the model and it is not so irrelevant to consider the specific variancecovariance structures to be defined for the two errors in a real context. In general, a diagonal structure could be assumed for the two matrices $\boldsymbol{G}$ and $\boldsymbol{R}$, that refers to a situation in which all the covariances are null. Nevertheless, when considering the split-plot design, the diagonal structure for both the $\boldsymbol{G}$ and $\boldsymbol{R}$ matrices is often misleading given that a covariance is present among the observations within the same WP unit.

In what follows, the joint split-plot model is explained (Section 2.4), and further considerations on the structure of the $\boldsymbol{G}$ and $\boldsymbol{R}$ matrices are detailed in the case-study (Section 3.1).

\subsection{The joint mixed split-plot model}

The fitting of only one statistical model for several $T$ responses is based on the definition of a new response variable $\boldsymbol{Y}$, built through the (vertical) concatenation of $T$ column vectors (related to the responses): $\boldsymbol{Y} \equiv\left[\boldsymbol{y}_{1}: \ldots .: \boldsymbol{y}_{T}\right]$. The new statistical model allows for a joint evaluation of variance and covariance parameters by considering the specific sources of variability: WP factors, SP factors, also conditioned by responses. All of these issues are a further improvement for the suggested optimization procedure. Therefore, the split-plot mixed RS model for a joint response $\boldsymbol{Y} \equiv\left[\boldsymbol{y}_{1}: \ldots: \boldsymbol{y}_{T}\right]$ is defined as follows:

$$
\boldsymbol{Y}=\dot{\boldsymbol{X}} \dot{\boldsymbol{\beta}}+\dot{Z} \dot{\boldsymbol{\gamma}}+\epsilon
$$


where:

1. $\dot{\boldsymbol{X}}$ is a model matrix consisting of the horizontal concatenation of four algebraic elements:

- the unit column vector $\mathbf{1}_{\mathbf{T N}}$ (length $T \times N$ ), used for estimating the intercept;

- a column vector $\boldsymbol{v}$ (length $T \times N$ ) defined to study the contribution of each specific response variable within the joint statistical model. In general, $\boldsymbol{v}$ is a column vector of length $T N$; for example, if we assume that there are two responses $(T=2), \boldsymbol{v}$ is a binary column vector with length $2 N$;

- a matrix $\tilde{\boldsymbol{X}}$ containing the set of column vectors for the estimates of 'common' fixed effects (without considering the specific differentiation by $\boldsymbol{y}_{t}$ );

- a matrix $\boldsymbol{X}(v)$ in which each column vector is defined for the estimation of a main effect or a first order interaction term conditioned by each response variable. It must be noted that this term corresponds to the matrix $\boldsymbol{X}_{t}$ defined in formulas (5) and (6).

Therefore, the structure of $\dot{\boldsymbol{X}}$ is the following:

$$
\dot{\boldsymbol{X}} \equiv[\mathbf{1}: \boldsymbol{v}: \tilde{\boldsymbol{X}}: \boldsymbol{X}(v)]
$$

The matrix $\dot{\boldsymbol{X}}$ has a general dimension: $[T N \times p]$ where $p=[1+T+\operatorname{noc} \operatorname{col}(\tilde{\boldsymbol{X}})+$ $\operatorname{no.col}(\boldsymbol{X}(v))]$;

2. $\dot{\boldsymbol{\beta}}$ is the global column vector relating to the unknown coefficients for the fixed part of the model corresponding to the matrix $\dot{\boldsymbol{X}}$; thus, $\dot{\boldsymbol{\beta}}$ is formed through the vertical concatenation of four column vectors and has the following structure:

$$
\dot{\boldsymbol{\beta}} \equiv\left[\boldsymbol{\beta}_{0}: \boldsymbol{\beta}_{v}: \tilde{\boldsymbol{\beta}}: \boldsymbol{\beta}(v)\right]
$$

where $\boldsymbol{\beta}_{0}$ involves the unknown parameters for the intercepts, $\boldsymbol{\beta}_{v}$ is the vector of coefficients for measuring the differences in responses, $\tilde{\boldsymbol{\beta}}$ is the column vector containing the unknown parameters for matrix $\tilde{\boldsymbol{X}}, \boldsymbol{\beta}(v)$ involves the unknown parameters corresponding to the effects defined through the matrix $\boldsymbol{X}(v)$; thus, $\dot{\boldsymbol{\beta}}$ has a general dimension equal to $[p \times 1]$;

3. $\dot{Z}$ is a matrix consisting of all the column vectors relating to the random effects, also including the random effects useful for measuring the robust design setting. Therefore, $\dot{Z}$ is the extension of $\dot{\boldsymbol{Z}}_{t}$ (formulas (5) and (6)) and it is formed by the horizontal concatenation of (at least) three matrices:

- $\tilde{Z}$ which contains vectors of random effects for the WP factors, including interactions and quadratic effects, and the random effect of replicates;

- $\boldsymbol{Z}(v)$ which includes the random effects conditioned by the response variables;

- $\boldsymbol{\Delta}$ is a matrix formed by the first order interaction terms related to noises and process variables.

Therefore, $\dot{Z}$ has the following structure:

$$
\dot{\boldsymbol{Z}} \equiv[\tilde{\boldsymbol{Z}}: \boldsymbol{Z}(v): \boldsymbol{\Delta}]
$$

The general dimension for the matrix $\dot{\boldsymbol{Z}}$ is equal to: $[T N \times h]$, where $h=[\operatorname{no} \cdot \operatorname{col}(\tilde{\boldsymbol{Z}})+$ $\operatorname{no.col}(\boldsymbol{Z}(v))+\operatorname{no.col}(\boldsymbol{\Delta})]$; 
4. $\dot{\gamma}$ is the global column vector defined as the vector of unknown parameters for $\dot{\boldsymbol{Z}}$; more specifically, $\dot{\gamma}$ includes the set of unknown random parameters for the WP structure $(\tilde{\gamma})$, the random effects estimated conditioned by each response variable $(\gamma(v))$; and the column vector of unknown parameters $\boldsymbol{\delta}$ related to the estimated coefficients for the robust design approach; thus, we obtain:

$$
\dot{\gamma} \equiv[\tilde{\gamma}: \gamma(v): \delta]
$$

where $\dot{\gamma}$ has a general dimension $[h \times 1]$.

It must be noted that the $\boldsymbol{\beta}(v)$ and the $\boldsymbol{\gamma}(v)$ are vectors of coefficients conditioned to each response variable and defined in order to detect the relation among the $T$ response variables in both the estimation and optimization steps.

As regards the variance-covariance structures, the matrix $G$ represents the variance-covariance matrix for random effects involved in model (7) through $\dot{Z}$; in general, for a split-plot design $\boldsymbol{G}$ could have a specific structure, such as variance components, compound symmetry or unstructured, relating to specific assumptions on correlations among random effects (WP factors). The matrix $\boldsymbol{R}$ is formed by the estimates of variance-covariance effects for the residual error $\boldsymbol{\epsilon}$, estimated for each replicate $k(k=1, . ., K)$ within each response variable $\left(\gamma_{k(v)}\right)$, and it could analogously assume a specific structure like matrix $\boldsymbol{G}$. For our case-study, we assume a compound symmetry structure for the matrix $\boldsymbol{G}$, and a diagonal structure for the matrix $\boldsymbol{R}$.

\subsection{The optimization step}

As also described in the introductive Section, one of the innovative contribution of this study is the joint optimization (in a multiple response case) by using an -only one- joint split-plot estimated model. When speaking about robust process optimization, we should consider the recent developments in this field starting from the Taguchi's two-step procedure (Nair 1992), and the seminal paper on the dual response approach by Vining and Myers (1990), where the expected value of the response (process variable) is optimized with respect to the objective value, also called the target $(\tau)$, and by simultaneously minimizing the process variability. Together with the dual response approach, the robust design concept was introduced by Taguchi in order to make insensitive a product (or a process) with respect to environmental noises and/or other internal or external sources of variability, see Nair (1992) for a detailed discussion of the Taguchi' method. Moreover, the achievement of a robust design is performed by setting the control factor levels to be insensitive to noise variables, often involved in the studies as random effects.

More recently, the robust design concept has been expounded involving the optimization step, in which noises and/or random effects are also evaluated (Berni and Burbui 2014; Berni and Bertocci 2018).

To this end, in this study, the joint estimated split-plot model is used to define the objective function to be optimized, by considering:

- the estimated fixed coefficients specifically related to each response $(\boldsymbol{\beta}(v))$, where each input variable, e.g. $x_{i}$ is crossed with $\boldsymbol{v}$;

- the estimated fixed 'common' coefficients $(\tilde{\boldsymbol{\beta}})$;

- the estimated coefficients that measure the relation among the responses, in general defined by the vector $\boldsymbol{\beta}_{v}$.

In addition the random effects are involved in the process optimization by including the estimated confidence interval for each random coefficient.

Therefore, and in order to evaluate all these model terms within the objective function, we 
consider to start from the estimation of the joint general mixed RS model (7) considering the $T$ dependent variables.

The simultaneous optimization is carried out by involving these $T$ estimated surfaces through the joint model (7). Furthermore we define $F_{t}$ for each $\boldsymbol{y}_{t}, t=1, \ldots, T$ (formula (12)), computed as the difference (quadratic distance) between the estimated surface $\hat{Y}_{t}$ through the joint model (formula (7)) and the target value $\tau_{t}$ a-priori specified for each response; therefore, the objective function is articulated as follows:

$$
\begin{array}{r}
F_{t}(Z, X)=\left(\hat{Y}_{t}(Z, X)-\tau_{t}\right)^{2} ; \forall t \\
\text { and } \\
\min _{(Z, X) \in \chi} \sum_{t} F_{t}(Z, X)
\end{array}
$$

It must be noted that formula (12) takes each response variable into account; then, by means of the second formula (13), the joint minimization is carried out within the coded experimental region $\chi$. All the random effects, estimated through the joint modeling (formula (7)), are also included, even though through the estimated confidence limits only. Moreover, through formula (12) we can perform the dual response approach (Vining and Myers 1990), in which the adjustment to the target value and the minimization of process variability are performed simultaneously.

By summarizing, the joint optimization is carried out by considering: i) fixed effects, e.g. the estimated coefficients of the global column vector $\dot{\boldsymbol{\beta}}$ defined in formula (9); ii) random effects through the estimated coefficients of the column vector $\dot{\gamma}$, formula (11), and the estimated confidence intervals. Moreover, constraints are included in order to keep the analytical optimization within the coded experimental region $\chi$, and avoid searching for optimum values without any accurate diagnostic checks.

\subsection{The full procedure for joint modeling and optimization}

Although the full procedure is implemented for several response variables, it is important to also perform a preliminary analysis for each response variable. Generally, in this preliminary analysis, for each $\boldsymbol{y}_{t},(t=1, . ., T)$, the distribution assumptions should be checked, and the experimental data, even though expressed with the same unit of measure, must be standardized.

By considering the modeling step (Section 2.4) and the subsequent optimization step (Section 2.5 ), the full procedure for a joint modeling and optimization may be summarized as follows:

1. Creation of the response variable $\boldsymbol{Y}$ as a vector of length equal to $N T=N \times T$; creation of a column vector $\boldsymbol{v}$ opportunely coded to account for coefficient estimates for each response within the joint model. If we assume that there are $T=2$ responses, then $\boldsymbol{v}$ reduces to a binary vector.

2. Estimation of the best fitted joint model, based on formula (7), by including the WP and/or SP variable effects crossed with $\boldsymbol{v}$ in order to evaluate the individual contribution of each response.

3. Definition of the objective function, as defined in formulas (12) and (13), and based on the estimated model (7). Each $F_{t}(Z, X), \forall t$, can be defined as the part of the objective function specifically related to the corresponding $\boldsymbol{y}_{t}$, by including common and specific fixed coefficients, and random effects. Each estimated random coefficient must be involved in the optimization step by including the corresponding confidence limits. Then, the objective function must be minimized (formula (13), Section 2.5).

We now verify the full procedure of a joint modeling and optimization by showing an empirical example involving experimental data planned through a split-plot design (Section 3). 


\section{A split-plot case-study on printed circuit boards}

The application relates to the microelectronics field in order to study the soldering performance of electronic components on printed circuit boards according to the specific materials used (Berni, Scarano, Bertocci, and Catelani 2013). More precisely, we study a split-plot design involving two quantitative response variables: i) pull-force level $[N]$, ii) electrical resistance $[\Omega]$. There are no specific targets for the two response variables $\boldsymbol{y}_{t},(T=2)$; we desire maximization for the pull-force level $\left(y_{1}\right)$ and a minimization for the electrical resistance $\left(y_{2}\right)$. Nevertheless, by considering the effective process and the targets effectively desirable and jointly achieved, a maximum coded value of $\tau_{1}$ greater than 1.008 and a minimum coded value of $\tau_{2}$ lower than 0.128 could be considered as a satisfactory result. Table 1 contains a summarized description of responses.

Table 1: Description of the response variables

\begin{tabular}{|llc|}
\hline Variables & Symbol & Target $\tau_{t}$ (coded) \\
\hline Pull-force level & $y_{1}$ & 1.008 \\
Electrical resistance & $y_{2}$ & 0.128 \\
\hline
\end{tabular}

Table 2: Description of the experimental factors and variables

\begin{tabular}{|lll|}
\hline Variables & Symbol & Coded Levels \\
\hline Printed Circuit Board-PCB (WP) & $z_{p c b, l}$ & $l=1,2,3,4$ \\
Time [h] (SP) & $x_{1}$ & {$[-1,1]$} \\
Pin Grid Array-PGA [pin size] (SP) & $x_{2}$ & {$[-1,1]$} \\
\hline
\end{tabular}

We consider four different motherboards, e.g. printed circuit boards - PCBs, each one characterized by a different surface finish and a specific soldering alloy. Each PCB is studied with respect to two internal components. Two integrated circuits (IC) are considered in each PCB; each IC is connected to the PCB by a different sized Pin Grid Array (PGA), also simply referred to as pin. In this study, two pin components are considered, and reported with coded levels for privacy reasons (Table 2). In addition, the time variable is studied at two levels in order to verify the soldering performance over time. Therefore, in this split-plot design, three variables are involved: four PCBs, two electronic components (characterized by a different sized pin) and time. In general the discrimination between WP and SP factors can be performed considering the process framework and/or the nature of experimental variables, especially when block or noises are present. In this case the discrimination has been done by considering that the PCB factor is primarily involved in the process, and it is the physical base for the electronic components, e.g. the PGA. Therefore, the PCB is considered as a WP factor at four levels $\left(z_{p c b, 1}\right)$, while time $\left(x_{1}\right)$ and components (pin size, $\left.x_{2}\right)$ are included in the experimental design as SP factors. Table 2 contains a description of the WP and SP experimental variables. Six replicates (blocks) are carried out, i.e. $K=6$. Thus, the final split-plot design has one WP factor (i.e. $I=1$ ) and two SP factors (i.e. $J=2$ ); each replicate $\boldsymbol{b}_{k}, k=1, . ., 6$, is formed by $n=4^{1} \times 2^{2}=16$ runs and the full design involves $N=n \times K=96$ observations, given by six replicates. It must be noted that both response variables are normalized according to the pin size of the components in order to estimate a joint statistical model but also to consider the different cumulate power due to the different pin size. The 
statistical analysis is carried out by applying the MIXED and NLP procedures of the SAS software (version 9.2, Windows Platform).

\subsection{Model and optimization results}

By following our proposal (Section 2.4) and in order to estimate the joint model, a single vector $\left(\boldsymbol{Y}=\left(y_{1}, \ldots y_{u}, \ldots y_{192}\right)\right)$ is built for the $T=2$ standardized response variables and a binary vector $(\boldsymbol{v})$ is included in the model. Given the specific nature of the experimental data, we estimate a mixed RS model where the one WP factor, e.g. PCBs, is included in the model as a fixed as well as a random effect. More specifically the PCB factor is considered as fixed main effect at four level (Table 3); moreover in order to evaluate the relevance of PCBs with respect to each response, we include a random coefficient $\gamma_{p c b(v), l}$ for each type of PCB by response (Table 4 ).

Table 3: GLS estimates for fixed effects of model (14)

\begin{tabular}{|llll|}
\hline Coeff.(effect) & estimate & st.err. & p-value \\
\hline$\beta_{0}$ & 0.8992 & 0.0385 & 0.0002 \\
$\beta_{v}($ response) & -0.0366 & 0.0593 & 0.5810 \\
$\beta_{v}(-)$ & 0 & - & - \\
$\tilde{\beta}_{11}\left(z_{p c b, 1}\right)$ & 0.0837 & 0.0263 & 0.0501 \\
$\tilde{\beta}_{12}\left(z_{p c b, 1}\right)$ & -0.0207 & 0.0263 & 0.4897 \\
$\tilde{\beta}_{13}\left(z_{p c b, 1}\right)$ & -0.0065 & 0.0263 & 0.8198 \\
$\tilde{\beta}_{14}\left(z_{p c b, 1}\right)$ & 0 & - & - \\
$\tilde{\beta}_{2}\left(x_{1}\right)$ & 0.1934 & 0.0189 & 0.0020 \\
$\tilde{\beta}_{3}\left(x_{2}\right)$ & -0.010 & 0.0052 & 0.0518 \\
$\beta_{41}\left(x_{1} v\right)$ & -0.0235 & 0.0582 & 0.6895 \\
$\beta_{42}\left(x_{1} v\right)$ & 0 & - & - \\
$\beta_{51}\left(x_{2} v\right)$ & 0.0216 & 0.0582 & 0.7114 \\
$\beta_{52}\left(x_{2} v\right)$ & 0 & - & - \\
\hline
\end{tabular}


Table 4: Estimates for random effects of model (14)

\begin{tabular}{|llll|}
\hline Coeff.(effect) & estimate & st.err. & p-value \\
\hline$\tilde{\gamma}_{12}$ & -0.1585 & 0.0052 & 0.0001 \\
$\tilde{\gamma}_{1}$ & -0.1534 & 0.0216 & 0.0001 \\
$\tilde{\gamma}_{2}$ & -0.1579 & 0.0195 & 0.0001 \\
$\tilde{\gamma}_{3}$ & -0.1547 & 0.0196 & 0.0001 \\
$\tilde{\gamma}_{4}$ & -0.1532 & 0.0196 & 0.0001 \\
$\tilde{\gamma}_{5}$ & -0.1572 & 0.0202 & 0.0001 \\
$\tilde{\gamma}_{6}$ & -0.1654 & 0.0201 & 0.0001 \\
$\gamma_{p c b(1), 1}$ & -0.1588 & 0.0227 & 0.0001 \\
$\gamma_{p c b(1), 2}$ & -0.1561 & 0.0227 & 0.0001 \\
$\gamma_{p c b(1), 3}$ & -0.1564 & 0.0227 & 0.0001 \\
$\gamma_{p c b(1), 4}$ & -0.1566 & 0.0227 & 0.0001 \\
$\gamma_{p c b(2), 1}$ & -0.1552 & 0.0227 & 0.0001 \\
$\gamma_{p c b(2), 2}$ & -0.1578 & 0.0227 & 0.0001 \\
$\gamma_{p c b(2), 3}$ & -0.1576 & 0.0227 & 0.0001 \\
$\gamma_{p c b(2), 4}$ & -0.1573 & 0.0227 & 0.0001 \\
$\delta_{1 p c b, 1}$ & -0.1295 & 0.0197 & 0.0001 \\
$\delta_{1 p c b, 2}$ & -0.1734 & 0.0197 & 0.0001 \\
$\delta_{1 p c b, 3}$ & -0.1662 & 0.0197 & 0.0001 \\
$\delta_{1 p c b, 4}$ & -0.1587 & 0.0197 & 0.0001 \\
\hline
\end{tabular}


Table 5: Estimated variance-covariance effects and Wald Z-test

\begin{tabular}{|llll|}
\hline Effect & REML estimate & s.e. & p-value \\
\hline$\sigma^{2}$ & $2.47 \mathrm{e}-4$ & $1.99 \mathrm{e}-4$ & 0.1069 \\
$\sigma_{C S}^{2}$ & 0.0249 & 0.0356 & 0.4843 \\
\hline$\gamma_{1(1)}$ & 0.3116 & 0.1110 & 0.0025 \\
$\gamma_{2(1)}$ & 0.3251 & 0.1155 & 0.0024 \\
$\gamma_{3(1)}$ & 0.3251 & 0.1154 & 0.0024 \\
$\gamma_{4(1)}$ & 0.3241 & 0.1151 & 0.0024 \\
$\gamma_{5(1)}$ & 0.3251 & 0.1154 & 0.0024 \\
$\gamma_{6(1)}$ & 0.3241 & 0.1151 & 0.0024 \\
$\gamma_{1(2)}$ & 0.0143 & 0.0052 & 0.0033 \\
$\gamma_{2(2)}$ & $1.168 \mathrm{e}-3$ & $6.55 \mathrm{e}-4$ & 0.0051 \\
$\gamma_{3(2)}$ & $1.884 \mathrm{e}-3$ & $7.86 \mathrm{e}-4$ & 0.0083 \\
$\gamma_{4(2)}$ & $1.884 \mathrm{e}-3$ & $8.20 \mathrm{e}-4$ & 0.0108 \\
$\gamma_{5(2)}$ & $3.543 \mathrm{e}-3$ & $1.41 \mathrm{e}-3$ & 0.0060 \\
$\gamma_{6(2)}$ & $3.272 \mathrm{e}-3$ & $1.276 \mathrm{e}-3$ & 0.0052 \\
\hline
\end{tabular}

According to formula (7), the split-plot model, expressed for the $u$-th trial and applied to the experimental data, is the following:

$$
\begin{array}{r}
y_{u}(Z, X)=\beta_{0}+\beta_{v} v_{u}+\sum_{l=1}^{L-1} \tilde{\beta}_{1, l} z_{p c b_{u, l}}+\tilde{\beta}_{2} x_{1 u}+\tilde{\beta}_{3} x_{2 u}+ \\
\sum_{s=1}^{2} \beta_{4, s} x_{1 u} v_{u}+\sum_{s=1}^{2} \beta_{5, s} x_{2 u} v_{u}+\tilde{\gamma}_{12} x_{1 u} x_{2 u}+ \\
\sum_{k=1}^{K=6} \tilde{\gamma}_{k} b_{u k}+\sum_{l=1}^{L-1} \gamma_{p c b(1), l} z_{p c b_{u, l}}+\sum_{l=1}^{L-1} \gamma_{p c b(2), l} z_{p c b_{u, l}}+ \\
x_{1 u} \sum_{l=1}^{L-1} \delta_{1 p c b, l} z_{p c b_{u, l}}+ \\
\sum_{k=1}^{K=6} \gamma_{k(1)} b_{u k}+\sum_{k=1}^{K=6} \gamma_{k(2)} b_{u k}
\end{array}
$$

In formula (14), $l=1, . ., 4$ denotes the levels for the WP factor (e.g. the PCBs) estimated as fixed effect, and as the random effect conditioned by responses $\left(\gamma_{p c b(v), l}\right)$, given the specific relevance of this process qualitative variable; $s=1,2$ is the number of coefficients to be estimated when considering the time and pin effects conditioned by each response variable. Therefore, the two terms $x_{1 u} v_{u}$ and $x_{2 u} v_{u}$ in model (14) are not first order interaction effects 
strictu sensu, instead they measure the SP variable effects of time $\left(x_{1}\right)$ and pin $\left(x_{2}\right)$ due to each response variable involved in the model estimation, e.g. the $\boldsymbol{X}(v)$ and $\boldsymbol{\beta}_{v}$ terms of formulas (8) and (9) respectively.

The matrix $\boldsymbol{G}$ of variance-covariance for random effects has a compound symmetry structure. Thus, by denoting the matrix $\left[\mathbf{1}_{h}^{\prime} \times \mathbf{1}_{h}\right]$ with $\boldsymbol{J}(h=19)$, the matrix $\boldsymbol{G}$ for the estimated model (14) is as follows:

$$
\boldsymbol{G}=\sigma^{2} \boldsymbol{I}_{h \times h}+\sigma_{C S}^{2} \boldsymbol{J}_{h \times h}=2.47 \mathrm{e}-4 \boldsymbol{I}_{h \times h}+0.0249 \boldsymbol{J}_{h \times h} .
$$

The variance-covariance effects for the residual errors are estimated for each replicate, within each response variable; each diagonal block matrix $\boldsymbol{R}_{k_{t}} ; k=1, . ., 6 ; t=1,2$ has dimension [16 $\times 16]$. In Table (5) we show the variance and covariance estimates for the random effects ( $\boldsymbol{G}$ and $\boldsymbol{R}$ estimates) estimated through the REML method (Section 2.1).

Furthermore, $\boldsymbol{R}$ is a diagonal matrix formed by twelve $\boldsymbol{R}_{k_{t}}$ blocks; each variance component is the diagonal element of the corresponding $k$ block $k=1, . ., 6 ; n_{k}=16 \forall k$, conditioned by each response variable. The assumed diagonal structure for the matrix $\boldsymbol{R}$ provides the best model fit for the data in our case-study. Following is the matrix $\boldsymbol{R}$ for the estimated model (14):

$$
\begin{aligned}
\boldsymbol{R} & =\left(\begin{array}{cccc}
\sigma_{1(1)}^{2} \boldsymbol{I}_{n_{16}} & \mathbf{0} & \ldots & \mathbf{0} \\
\mathbf{0} & \sigma_{2(1)}^{2} \boldsymbol{I}_{n_{16}} & \ldots & \mathbf{0} \\
\vdots & \vdots & \ddots & \\
\mathbf{0} & \mathbf{0} & \ldots & \sigma_{6(2)}^{2} \boldsymbol{I}_{n_{16}}
\end{array}\right) \\
& =\left(\begin{array}{ccccc}
0.3116 \boldsymbol{I}_{n_{16}} & \mathbf{0} & \ldots & \mathbf{0} \\
\mathbf{0} & 0.3251 \boldsymbol{I}_{n_{16}} & \ldots & \mathbf{0} \\
\vdots & \vdots & & \vdots \\
\mathbf{0} & \mathbf{0} & \ldots & 3.272 \mathrm{e}-3 \boldsymbol{I}_{n_{16}}
\end{array}\right)
\end{aligned}
$$

We do not report the correlation matrix of the estimates: the maximum absolute value is 0.2956 ; the minimum is $1.6 \mathrm{e}-4$. Moreover, the likelihood ratio test statistic is equal to 187.60 $(\mathrm{df}=13)$ with a highly significant $\mathrm{p}$-value $(p<0.0001)$.

In Table 3 we show the GLS estimates for model (14). By observing Table 3, we note that the WP factor, e.g. PCBs, is not significant in two out of the three coefficients estimated with respect to the fourth PCB type (reference level estimation). Nevertheless, it is relevant to include this factor also as a fixed effect to evaluate the performance of each PCB in the following optimization step. Moreover, the estimated coefficient related to the response variables $\left(\beta_{v}\right)$ is not significant; while the two SP factors, e.g. pin and time, are significant (time is highly significant) as linear effects, they are not significant when considering the differences by the responses. The estimated coefficients, e.g. $\beta_{41} \beta_{42} \beta_{51}$ and $\beta_{52}$, are included in the model in order to evaluate the specific contribution of each SP variable $\left(x_{1}, x_{2}\right)$ to each response, and in relation to the other one. In fact, these two interaction terms are calculated by crossing each SP variable with the vector $\boldsymbol{v}$, defined in Section 2.4.

The replicate effects are involved in model $(14)$ as random effects $\left(\tilde{\gamma}_{k} ; k=1, . .6\right)$ and through the estimation of the corresponding variance-covariances effects by response variables (Table 5), as explained previously. Furthermore, two other random effects are considered: i) the first order interaction for the two SP variables, pin and time $\left(\tilde{\gamma}_{12}\right)$; ii) the first order interaction between the SP variable time and the WP factor PCBs, estimated through the coefficient $\delta_{1 p c b, l}, l=1, . ., 4$.

Table 4 illustrates the estimates for random effects involved in model (14). It must be noted that we apply $v=1,2$ to differentiate the estimates by response in model (14) and in Tables 4 and 5 . 
When observing Table (4), the estimated standard errors for the $\hat{\gamma}_{p c b(s), l}$ and $\hat{\delta}_{p c b, l}$ coefficients are equal; this issue can be explained by considering the specific definition of the errors in a compound symmetry structure and a split-plot balanced situation (Littell, Pendergast, and Natarajan 2000).

When considering Section 2.4 and the statistical model (7), we have the following algebraic structures:

1.

$$
\dot{\boldsymbol{X}} \equiv[\mathbf{1}: \boldsymbol{v}: \tilde{\boldsymbol{X}}: \boldsymbol{X}(v)]
$$

with specific dimension: $[T N \times p]=[2(96) \times 13]$ and $p=[1+T+\operatorname{no} \cdot \operatorname{col}(\tilde{\boldsymbol{X}})+\operatorname{no} \cdot \operatorname{col}(\boldsymbol{X}(v))]=[1+$ $2+6+4]$

2.

$$
\dot{\boldsymbol{\beta}} \equiv\left[\boldsymbol{\beta}_{0}: \boldsymbol{\beta}_{v}: \tilde{\boldsymbol{\beta}}: \boldsymbol{\beta}(v)\right]
$$

with specific dimension $\dot{\boldsymbol{\beta}}=[p \times 1]=[13 \times 1], p=13$;

3.

$$
\dot{\boldsymbol{Z}} \equiv[\tilde{\boldsymbol{Z}}: \boldsymbol{Z}(v): \boldsymbol{\Delta}]
$$

with specific dimension $[T N \times h]=[2(96) \times 19]$; it must be noted that the number of columns for $\boldsymbol{Z}(v)$ are eight in this example; moreover, $\tilde{\boldsymbol{Z}}$ has seven columns (six columns for the replicate effects and one column for the interaction between pin and time); $\boldsymbol{\Delta}$ has four columns, due to the first order interaction between time and PCBs;

4 .

$$
\dot{\gamma} \equiv[\tilde{\gamma}: \gamma(v): \delta]
$$

$\dot{\gamma}$ includes $h=19$ estimated coefficients related to all the random effects: seven belong to the $\tilde{\gamma}$ column vector, eight coefficients are contained in the $\gamma(v)$ vector and four are included in vector $\boldsymbol{\delta}$;

5. the matrix $\boldsymbol{G}$ of variance and covariance for random effects has a dimension of [19 $\times 19]$;

6. the matrix $\boldsymbol{V}$ for the joint response $\boldsymbol{Y}$ has the following framework:

$$
V=Z G Z^{\prime}+R
$$

and dimension:

$$
\begin{aligned}
\boldsymbol{V} & =[N \times N]=[N \times h] \times[h \times h] \times[h \times N]+[N \times N] \\
& =[192 \times 192]=[192 \times 19] \times[19 \times 19] \times[19 \times 192]+[192 \times 192] .
\end{aligned}
$$

When considering formula (13) and the optimization step, the estimated model reported in formula (14) is split into two functions $(T=2)$, according to the estimated coefficients for the fixed and random effects reported in Tables 3, 4 and 5. The estimates of variance-covariances for error and random effects (Tables 4 and 5) are included in the subsequent optimization step in order to evaluate the variability linked to the full process and planning, and also for the robust design situation. All the random coefficients are involved in formula (12) by imposing the corresponding confidence limits, while for the fixed effects the $[\min , \max ]$ range on $\chi$ is considered. 
Table 6: Optimization results by PCB; formula (13)

\begin{tabular}{|ll|}
\hline Results & $P C B_{1}$ \\
\hline Targets & $\hat{\tau_{1}}=1.006 ; \hat{\tau_{2}}=4.4 e-4$ \\
Variables & $x_{1}=1 ; x_{2}=-0.44$ \\
Diagnostics & \\
of; $|H|$ & $0.9815 ;>1.85 \mathrm{e}-10$ \\
\hline Results & $P C B_{2}$ \\
\hline Targets & $\hat{\tau_{1}}=1.006 ; \hat{\tau_{2}}=4.4 e-4$ \\
Variables & $x_{1}=1 ; x_{2}=0.36$ \\
Diagnostics & \\
of; $|H|$ & $1.335 ; 4.7 e-15$ \\
\hline Results & $P C B_{3}$ \\
\hline Targets & $\hat{\tau_{1}}=1.006 ; \hat{\tau_{2}}=4.4 e-4$ \\
Variables & $x_{1}=1 ; x_{2}=0.36$ \\
Diagnostics & \\
of; $|H|$ & $1.271 ;>1.11 e-15$ \\
\hline Results & $P C B_{4}$ \\
\hline Targets & $\hat{\tau_{1}}=1.006 ; \hat{\tau_{2}}=4.4 e-4$ \\
Variables & $x_{1}=1 ; x_{2}=0.50$ \\
Diagnostics & \\
of; $|H|$ & $1.216 ;>1.0 e-10$ \\
\hline
\end{tabular}

Target values are recalculated on standardized data, so that the desired target values are greater than the lower value $\tau_{1}=1.008$ for the pull-force level and lower than the upper value $\tau_{2}=0.128$ for electrical resistance (Table 1). Therefore the optimal solution, e.g. the best factor values combination, is identified according to the fixed target values, although anything greater for the pull-force level and lower for the electrical resistance would be even better. Furthermore, when considering the time variable, we assume that an optimal time solution is obtained when the time is greater than 0.1870 , corresponding to 2500 cycles.

The optimization results are reported in Table 6 . It must be noted that the optimization is carried out for each type of PCB separately, although it is performed for the two response variables jointly. For all the four optimizations per PCB type, we achieve convergency and good diagnostics for the objective function value $(o f)$, the absolute maximum gradient value $\left(\|x\|_{\infty}\right)$, and the determinant of the Hessian matrix $(|H|)$. Target values are always achieved, especially for the second response variable, the electrical resistance, which always obtains the very small value of 0.00044 . 
Nevertheless, together with the achievement of the target values, it is relevant to control for the time threshold: for all the PCBs, time achieves the upper coded limit equal to 1 . When considering the PGA variable, a different setting is achieved for each PCB. For privacy reasons, we report the coded results by PCB: the minimum pin size is -0.44 pin for PCBno.1, while the highest is achieved for $P C B n o .4$ with pin size equal to 0.50 . An equivalent behaviour in optimization is obtained for PCBno.2 and PCBno.3, where the pin size is fixed at 0.36 pin.

\section{Final remarks}

This paper attempts to improve the analysis of a split-plot design in a multiresponse situation by considering the mixed RS models and the robust design theory. These results are satisfactory when considering: i) the joint modeling of several responses; ii) the optimization carried out through a single measure which may also be differentiated by the responses; iii) the inclusion of random effects and errors in the optimization step.

Furthermore, it is worth mentioning the characteristics of the response variables involved in the joint modeling step. Nevertheless, the building of the joint model should take into account the presence of any correlation among responses, if it exists; even though the estimation of conditioned coefficients gives a measurement of the relation among the response variables.

\section{Acknowledgements}

Our thanks to Ms. Susan Mary Cadby for her revision of the English language aspects of the paper.

\section{References}

Berni R (2012). "Split-plot Design and Mixed Response Surface Models." In ENBIS-12 Programme and Abstracts of the 12th Annual Conference of the European Network for Business and Industrial Statistics (ENBIS), University of Ljubljana, 9-13 September 2012, pp. 1-4. ISBN 1-59593-322-0. URL https://books . google.it/books?id=UYFhIQEACAAJ.

Berni R, Bertocci F (2018). "Optimization of Gas Sensors Based on Advanced Nanomaterials through Split-Plot Designs and GLMMs." Sensors, 18(11), 3858-3870. doi:10.3390/ s18113858.

Berni R, Burbui M (2014). "Process Optimization of a Super-finishing Machine through Experimental Design and Mixed Response Surface Models." Quality Engineering, 26(4), 404-415. doi:10.1080/08982112.2013.872794.

Berni R, Scarano VL, Bertocci F, Catelani M (2013). "Mixed Response Surface Models and Bayesian Analysis of Variance Components for Electrically Conductive Adhesives." Applied Stochastic Models in Business and Industry, 29(4), 387-398. doi:10.1002/asmb.1978.

Bingham D, Nair VN (2012). "Noise Variable Settings in Robust Design Experiments." Technometrics, 54(4), 388-397. doi:10.1080/00401706.2012.694787.

Box GEP, Jones S (1992). "Split-plot Designs for Robust Product Experimentation." Journal of Applied Statistics, 19(1), 3-26. doi:10.1080/02664769200000001.

Del Castillo E (2007). Process Optimization: A Statistical Approach. Springer-Verlag New York. ISBN 978-0-387-71435-6.

Del Castillo E, Colosimo BM, Alshraideh H (2012). "Bayesian Modeling and Optimization of Functional Responses Affected by Noise Factors." Journal of Quality Technology, 44(2), 117-135. doi : 10.1080/00224065.2012.11917888. 
Derringer G, Suich R (1980). "Simultaneous Optimization of Several Response Variables." Journal of Quality Technology, 12(4), 214-219. doi:10.1080/00224065.1980.11980968.

Goos P, Gilmour SG (2012). "A General Strategy for Analyzing Data from Split-plot and Multistratum Experimental Design." Technometrics, 54(4), 340-354. doi:10.1080/00401706. 2012.694777.

Goos P, Gilmour SG (2017). "Testing for Lack of Fit in Blocked, Split-plot, and Other Multi-stratum Designs." Technometrics, 49(4), 320-336. doi:10.1080/00224065.2017. 11918000.

Ivanova A, Molenberghs G, Verbeke G (2019). "Mixed Models Approaches for Joint Modeling of Different Types of Responses." Journal of Biopharmaceutical Statistics, 26(4), 601-618. doi:10.1080/10543406.2015.1052487.

Jones B, Goos P (2012). "An Algorithm for Finding D-efficient Equivalent-estimation Secondorder Split-plot Designs." Journal of Quality Technology, 44(4), 363-374. doi:10.1080/ 00224065.2012 .11917906$.

Khuri AI (1996). "Response Surface Models with Mixed Effects." Journal of Quality Technology, 28(2), 177-186. doi:10.1080/00224065.1996.11979658.

Khuri AI (2006). "Mixed Response Surface Models with Heterogeneous within-block Error Variances." Technometrics, 48(2), 206-218. doi:10.1198/004017005000000599.

Khuri AI, Conlon M (1981). "Simultaneous Optimization of Multiple Responses Represented by Polynomial Regression Functions." Technometrics, 23(4), 363-375. doi: 10.1080/00401706.1981.10487681.

Khuri AI, Mukhopadhyay S (2010). "Response Surface Methodology." WIREs Computational Statistics, 2(2), 128-149. doi:10.1002/wics.73.

Krueger DC, Montgomery DC (2014). "Modeling and Analyzing Semiconductor Yield with Generalized Linear Mixed Models." Applied Stochastic Models in Business and Industry, 6(6), 691-707. doi:10.1002/asmb. 2074.

Lee DH, Kim KJ, Köksalan M (2012). "An Interactive Method to Multiresponse Surface Optimization Based on Pairwise Comparisons." IIE Transactions, 44(1), 13-26. doi: 10.1080/0740817X.2011.564604.

Lin CY (2018). "Robust Split-plot Designs for Model Misspecification." Journal of Quality Technology, 50(1), 76-87. doi:10.1080/00224065.2018.1404325.

Lin DKJ, Tu W (1995). "Dual Response Surface Optimization.” Journal of Quality Technology, 27(1), 34-39. doi : 10.1080/00224065.1995.11979556.

Littell RC, Pendergast J, Natarajan R (2000). "Modelling Covariance Structure in the Analysis of Repeated Measures Data." Statistics in Medicine, 19(13), 1793-1819. doi:10.1002/ 1097-0258(20000715) 19:13<1793: :AID-SIM482>3.0.CO;2-Q.

Logothetis N, Wynn HP (1989). Quality through Design- Experimental Design, Off-line Quality Control and Taguchi's Contributions. Clarendon Press, Oxford, UK. ISBN 9780198519935.

Medlin MR, Freeman LJ, Kensler JLK, Vining GG (2019). "Analysis of Split-plot Reliability Experiments with Subsampling." Quality and Reliability Engineering International, 35(3), 738-749. doi:10.1002/qre.2394. 
Myers RH, Montgomery DC (2002). Response Surface Methodology: Process and Product Optimization Using Designed Experiments. John Wiley \& Sons, 2nd ed, New York. ISBN 0471412554 .

Mylona K, Goos P, Jones B (2014). "Optimal Design of Blocked and Split-plot Experiments for Fixed Effects and Variance Component Estimation." Technometrics, 56(2), 132-144. doi:10.1080/00401706.2013.818579.

Nair VN (1992). "Taguchi's Parameter Design: A Panel Discussion." Technometrics, 30(4), 127-161. doi:10.2307/1269231.

Ng SH (2010). "A Bayesian Model-averaging Approach for Multiple-Response Optimization." Journal of Quality Technology, 42(1), 52-68. doi:10.1080/00224065.2010.11917806.

Robinson TJ, Pintar AL, Anderson-Cook CM, Hamada SM (2012). "A Bayesian Approach to the Analysis of Split-plot Combined and Product-arrays and Optimization in Robust Parameter Design." Journal of Quality Technology, 44(4), 304-320. doi: 10.1080/00224065.2012.11917903.

Searle SR, Casella G, McCulloch CE (1992). Variance Components. John Wiley \& Sons, New Jersey. ISBN 0471621625.

Tan MHY, Wu CFJ (2013). "A Bayesian Approach for Model Selection in Fractionated Splitplot Experiments with Application in Robust Parameter Design." Technometrics, 55(3), 359-372. doi:10.1080/00401706.2013.778790.

Tang LC, Xu K (2002). "A Unified Approach for Dual Response Surface Optimization." Journal of Quality Technology, 34(4), 437-447. doi:10.1080/00224065.2002.11980175.

Trinca LA, Gilmour SG (2017). "Split-plot and Multistratum Designs for Statistical Inference." Technometrics, 59(4), 446-457. doi:10.1080/00401706.2017.1316315.

Vining GG, Kowalski S, Montgomery DC (2005). "Response Surface Designs within a Splitplot Structure." Journal of Quality Technology, 37(2), 115-129. doi:10.1080/00224065. 2005.11980310.

Vining GG, Myers RH (1990). "Combining Taguchi and Response Surface Philosophies: A Dual Response Approach." Journal of Quality Technology, 22(1), 38-45. doi:10.1080/ 00224065 . 1990.11979204. 


\section{Affiliation:}

Rossella Berni

Department of Statistics Computer Science Applications 'G.Parenti'

University of Florence

Viale Morgagni 59, 50134 Florence, Italy

E-mail: rossella.berni@unifi.it

URL: https://www. unifi.it/p-doc2-2019-0-A-2b333a2a342c-1.html

Nedka Dechkova Nikiforova

Department of Statistics Computer Science Applications 'G.Parenti'

University of Florence

Viale Morgagni 59, 50134 Florence, Italy

E-mail: n.nikiforova@unifi.it

\section{Austrian Journal of Statistics}

published by the Austrian Society of Statistics

Volume 51

January 2022 http://www .ajs.or.at/

http://www.osg.or.at/

Submitted: 2020-08-04

Accepted: 2021-01-15 